\title{
Adaptação à prótese auditiva em usuários assistidos pelo Sistema Único de Saúde
}

\author{
Hearing aid adaptation in users assisted by the Unified Health \\ System
}

Ana Karina Lima Buriti ${ }^{1}$, Simone Helena dos Santos Oliveira ${ }^{2}$

\begin{abstract}
RESUMO
Objetivo: Avaliar a adaptação à prótese auditiva pelos usuários assistidos pelo Sistema Único de Saúde (SUS) e propor ações educativas em serviço, com base nas necessidades dos usuários. Métodos: Pesquisa descritiva, de natureza quantitativa, em que foram avaliados 32 adultos com deficiência auditiva, em fase de adaptação à prótese auditiva, que receberam pelo SUS. Foram aplicados dois questionários estruturados com questões fechadas, sendo realizada a análise descritiva dos dados. Resultados: Verificaram-se limitações e restrições moderadas e tempo reduzido de uso diário, dificultando o alcance do benefício do uso da amplificação sonora. Em relação ao uso e manuseio, foi observado que 70,8\% coloca e retira o aparelho sozinho, 79,2\% retira-o para tomar banho, 87,5\% não realiza a higienização diariamente e 37,5\% apresenta alergia ou dor atribuída ao uso do molde. Conclusão: Os usuários apresentam dificuldades em relação ao uso e ao manuseio da prótese, as quais influenciam negativamente na adaptação ao mesmo. Mostra-se necessária a promoção de ações de educação em saúde auditiva, a fim de minimizar tais dificuldades e favorecer a adaptação ao uso de prótese.
\end{abstract}

Descritores: Audição; Auxiliares de audição; Adaptação; Perda auditiva; Educação em saúde; Sistema único de saúde

\section{INTRODUÇÃO}

A audição é um dos sentidos mais importantes do ser humano para o desenvolvimento da comunicação oral. É através dela que ouvimos os sons do nosso ambiente e desenvolvemos as habilidades cognitivas e psicossociais. A privação auditiva, chamada de deficiência auditiva, incapacita o indivíduo a desempenhar essas habilidades. A deficiência auditiva nas crianças dificulta o desenvolvimento da linguagem, e nos idosos provoca o isolamento, diminuindo sua capacidade de comunicação e interação social ${ }^{(1)}$.

Segundo o Ministério de Saúde ${ }^{(2)}$, a deficiência passou a ser compreendida como parte ou expressão de uma condição de saúde, estabelecendo a interação entre a pessoa com deficiência, a limitação da atividade e os fatores do contexto sócio-ambiental.

Trabalho realizado em um serviço de reabilitação auditiva do Programa de Saúde Auditiva de alta complexidade do Hospital General Edson Ramalho (HPM) - João Pessoa (PB), Brasil.

(1) Programa de Pós-Graduação em Enfermagem (Mestrado), Universidade Federal da Paraíba - UFPB - João Pessoa (PB), Brasil.

(2) Universidade Federal da Paraíba - UFPB - João Pessoa (PB), Brasil. Endereço para correspondência: Ana Karina Lima Buriti. R. Severino Massa Spinelli, 200/1701, Tambaú, João Pessoa (PB), Brasil, CEP: 58039-210. E-mail: anak_buriti@yahoo.com.br Recebido em: 25/5/2011; Aceito em: 24/8/2011
Deste modo, foi instituída no Brasil em 28 de setembro de 2004, a Política Nacional de Atenção a Saúde Auditiva e as Portarias SAS/MS n ${ }^{\circ} 587$ e 589, que propõem a organização de uma rede hierarquizada, regionalizada e integrada na atenção básica, de média e alta complexidade para atendimento ao portador de deficiência auditiva ${ }^{(2)}$.

Após o diagnóstico e a indicação do uso do Aparelho de Amplificação Sonora Individual (AASI) pelo médico otorrinolaringologista, o fonoaudiólogo é responsável por selecionar o modelo e o tipo, mais adequados ao indivíduo com deficiência auditiva, de acordo com suas necessidades e prioridades para minimizar os danos da privação social ${ }^{(3)}$. Portanto, é fundamental esclarecer ao indivíduo a fase de adaptação da prótese auditiva e as etapas de (re)habilitação, garantindo melhor entendimento quanto aos benefícios, cuidados, manuseios, inserção e expectativas do indivíduo ao seu uso(4).

Vale ressaltar que a fase de adaptação é influenciada pelo uso e manuseio do AASI e que o indivíduo idoso apresenta dificuldades no manuseio do AASI, principalmente, na inserção e remoção do molde auricular. Os idosos também têm dificuldade no uso da tecnologia, em relação à utilização da bobina telefônica ou até mesmo das memórias do AASI, quando a tecnologia é digital; quando a tecnologia é analógica, o indivíduo apresenta outro tipo de dificuldade, relacionada às interferências da tecnologia ${ }^{(5)}$.

Pesquisa relacionada ao uso e manuseio da prótese auditiva 
em fase de adaptação revela que $12,90 \%, 58,06 \%$ e $67,74 \%$ dos indivíduos investigados apresentaram dificuldades relacionadas às pilhas, aos moldes ou cápsulas auriculares e às características da amplificação, respectivamente ${ }^{(6)}$.

Fundamentada nesse entendimento de cuidados com o AASI, que abrange tanto os cuidados de adaptação às características eletroacústicas programáveis pelo profissional, como os cuidados no uso e manuseio do AASI, cujas orientações devem ser fornecidas pelo mesmo profissional que o acompanha na fase de seleção e adaptação da prótese auditiva, considera-se relevante a temática acerca da saúde auditiva dos usuários do Sistema Único de Saúde (SUS), conforme os objetivos da Política Nacional de Atenção Saúde Auditiva, tendo em vista as características sócio-econômicas. Portanto, percebe-se uma grande dificuldade de manuseio e adaptação do AASI.

Diante do exposto, os objetivos desta pesquisa foram avaliar a adaptação de AASI em usuários assistidos pelo Sistema Único de Saúde e propor ações educativas em serviço, com base nas necessidades dos usuários da amplificação sonora assistidos pelo Sistema Único de Saúde.

\section{MÉTODOS}

Foram seguidas as recomendações da Resolução $\mathrm{n}^{\circ}$ 196/96 no concernente ao esclarecimento dos usuários acerca da pesquisa, do direito de recusar sua participação e de retirar-se da mesma a qualquer momento e sem nenhum ônus, assim como ao sigilo acerca da sua identidade, sendo assinado o Termo de Consentimento Livre e Esclarecido em duas vias. Antes do início da coleta de dados, a pesquisa foi autorizada pela instituição e aprovada pelo Comitê de Ética e Pesquisa do Hospital Universitário Lauro Wanderley, sob protocolo 309/2010.

$\mathrm{O}$ estudo foi realizado em um complexo hospitalar do município de João Pessoa (PB), onde existe um Serviço de Reabilitação Auditiva, vinculado ao Programa de Atenção a Saúde Auditiva (Portaria SAS/MS no 587, de 07/10/2004). A população do estudo foi constituída por 449 usuários que se beneficiaram da tecnologia do AASI no ano de 2009 pelo Sistema Único de Saúde (SUS) no município referido. Do total de usuários cadastrados, participaram do estudo adultos com deficiência auditiva em fase de adaptação a prótese auditiva. Foi considerado como critério de inclusão, o tempo de uso do AASI de pelo menos seis meses, concordância voluntária de participação na pesquisa, após a explicitação dos objetivos e métodos da mesma e da assinatura do Termo de Consentimento Livre e Esclarecido. Foram excluídos as crianças e adolescentes, adultos sem linguagem oral, incapazes de responder e compreender o questionário adequadamente, além dos usuários que não compareceram ao serviço no período da pesquisa e os que não concordaram em participar da pesquisa, obtendo-se uma amostra constituída por 32 usuários, sendo 17 mulheres e 15 homens, com idade entre 21 e 95 anos.

A coleta de dados foi realizada no período de março a maio de 2010, mediante a aplicação de dois questionários: o primeiro contendo questões relativas ao perfil sócio-demográfico, ao uso e manuseio das pilhas, dos moldes auriculares ou cápsula, e cuidados com o circuito da prótese, e o segundo, o Internatio- nal Outcome Inventory for Hearing Aids (IOI-HA), que é um instrumento incluído no Formulário de Seleção e Adaptação de Aparelhos de Amplificação Sonora Individual da Portaria SAS/MS n ${ }^{\circ}$ 587, de 07/10/2004 ${ }^{(7)}$.

O International Outcome Inventory for Hearing Aids (IOI-HA) foi traduzido por pesquisadores brasileiros ${ }^{(7)}$, sendo desenvolvido para realizar medidas de auto-avaliação em reabilitação auditiva, ou seja, tem o objetivo de medir a adaptação do AASI.

O IOI-HA é composto por oito questões com poucas exigências cognitivas para os indivíduos. Uma questão serve para estimar o grau de dificuldade auditiva que o indivíduo apresenta sem o uso dos AASI, e sete questões avaliam, de forma subjetiva, o resultado da adaptação ao AASI, sendo uma em cada um dos seguintes aspectos: benefício, limitação residual de atividades, satisfação, restrição residual de participação, impacto em outros e qualidade de vida.

O questionário oferece cinco opções de respostas graduadas da esquerda para a direita, de forma que a primeira opção refere-se a um pior desempenho, recebendo um ponto; a última opção indica um melhor desempenho, recebendo cinco pontos.

Após a aplicação dos questionários, dos 32 usuários de prótese auditiva que participaram da pesquisa, oito não participaram da aplicação do primeiro questionário porque não compareceram na segunda etapa do estudo. Foram, portanto, analisadas as respostas de 24 usuários aos dois questionários e da totalidade dos pesquisados apenas ao IOI-HA.

Os resultados de ambos os questionários foram analisados a partir da estatística descritiva (números absolutos e percentuais, média e desvio padrão) e apresentados em tabelas, sendo analisados à luz da literatura pertinente.

\section{RESULTADOS}

A faixa etária dos usuários que responderam aos dois questionários (24) foi variável, sendo que 8,3\% tinham entre 21 e 40 anos, $16,7 \%$ entre 41 e 60 anos, $50 \%$ entre 61 e 80 anos e $25 \%$ entre 81 e 100 anos. Quanto ao gênero, participaram $34,38 \%$ mulheres e $65,63 \%$ homens. Considerando o modelo e a tecnologia da prótese auditiva, apenas $12,5 \%$ utilizavam prótese auditiva digital programável intracanal e $87,5 \%$ utilizavam próteses retroauricular digital programável $(38,1 \%)$ e não programável (61,9\%).

Ao analisar os itens relacionados ao manuseio do AASI, verifica-se que a maioria dos usuários não apresenta dificuldades quanto à colocação e a retirada do AASI nem ao ajuste da pilha. Importa ressaltar que $70,8 \%$ dos indivíduos referem colocar o aparelho sozinhos (Tabela 1).

A Tabela 2 mostra a média e o desvio padrão referentes à pontuação total no questionário IOI-HA, aplicado aos usuários de AASI após pelo menos seis meses de adaptação. Os valores são apresentados pela média da somatória dos pontos (ST) obtidos em cada uma das sete questões. Assim, a pontuação total mínima é de um e máxima de cinco pontos. Uma maior pontuação indica melhores resultados em relação à adaptação do AASI.

$\mathrm{Na}$ Tabela 2, podemos observar que a maioria das médias para cada item analisado se situou pouco acima de três, o que 
Tabela 1. Dificuldades em relação ao manuseio e cuidados com a prótese auditiva

\begin{tabular}{|c|c|c|c|c|}
\hline \multirow{2}{*}{ Variáveis } & \multicolumn{2}{|c|}{$\operatorname{Sim}$} & \multicolumn{2}{|c|}{ Não } \\
\hline & $\mathrm{n}$ & $\% *$ & $\mathrm{n}$ & $\%^{*}$ \\
\hline \multicolumn{5}{|l|}{ Manuseio da prótese auditiva } \\
\hline Dificuldade em identificar a prótese da orelha esquerda e direita & 11 & 45,8 & 13 & 54,2 \\
\hline Coloca/retira a prótese auditiva sozinho & 17 & 70,8 & 7 & 29,2 \\
\hline Retira a pilha quando não estiver usando a prótese & 16 & 66,7 & 8 & 33,3 \\
\hline Abre/fecha o lugar onde fica a pilha & 19 & 79,2 & 5 & 20,8 \\
\hline Troca a pilha sem ajuda & 20 & 83,3 & 4 & 16,7 \\
\hline Identifica quando acaba a pilha da sua prótese auditiva & 15 & 62,5 & 9 & 37,5 \\
\hline Utiliza a bobina telefônica para falar ao telefone & 0 & 0,0 & 24 & 100 \\
\hline \multicolumn{5}{|l|}{ Cuidados com a prótese auditiva } \\
\hline Retira a prótese auditiva para tomar banho & 19 & 79,2 & 05 & 20,8 \\
\hline Tem cuidado para não derrubar sua prótese auditiva & 23 & 95,8 & 01 & 4,2 \\
\hline Limpa o aparelho diariamente & 06 & 25 & 18 & 75 \\
\hline \multicolumn{5}{|l|}{ Cuidados com o molde } \\
\hline Empresta-se o molde ou cápsula da sua prótese auditiva & 01 & 4,2 & 23 & 95,8 \\
\hline Tem conhecimento que deve ser feito o molde ou a cápsula anualmente & 03 & 12,5 & 21 & 87,5 \\
\hline O molde já causou alergia ou dor (machucando/inflamando) & 09 & 37,5 & 15 & 62,5 \\
\hline Lava o molde diariamente & 03 & 12,5 & 21 & 87,5 \\
\hline Sabe lavar seu molde & 02 & 8,3 & 22 & 91,7 \\
\hline Retira/coloca o molde da prótese auditiva & 16 & 66,7 & 08 & 33,3 \\
\hline
\end{tabular}

*: Os valores percentuais foram obtidos do número total de 24 usuários do SUS

Tabela 2. Adaptação à amplificação sonora, segundo os componentes do questionário IOI-HA

\begin{tabular}{lcc}
\hline Variáveis & Média $(\mathrm{x})$ & $\mathrm{DP}(\mathrm{s})$ \\
\hline Uso diário & 3,13 & 1,191 \\
Benefício & 2,88 & 1,116 \\
Limitações & 2,71 & 0,908 \\
Satisfação & 3,25 & 1,225 \\
Restrições & 3,50 & 1,351 \\
Convívio social & 3,50 & 1,251 \\
Qualidade de vida & 3,38 & 1,173 \\
\hline
\end{tabular}

não pode ser considerado satisfatório, pois revelam adaptação pouco positiva à amplificação sonora.

\section{DISCUSSÃO}

A partir dos dados evidencia-se que a grande maioria dos usuários de AASI deste estudo tem idade superior a 60 anos, aspecto esperado, pois uma das causas da diminuição da acuidade auditiva é o envelhecimento ${ }^{(1,8)}$.

No concernente ao manuseio do AASI, resultados semelhantes à nossa pesquisa foram encontrados em estudo com amostra semelhante, em que $72 \%$ dos usuários faziam colocação do aparelho sem dificuldades e $28 \%$ referiram que precisavam de ajuda para isso ${ }^{(6)}$.

Considerando que a maioria dos usuários de nossa pesquisa apresenta idade superior a 61 anos, foi satisfatório o manuseio da prótese auditiva sem dificuldade, uma vez que estes usuá- rios mantém preservada suficiente coordenação motora para o uso do AASI, favorecendo sua independência na decisão dos momentos de colocá-lo e de retirá-lo, de acordo com a sua necessidade e desejo de ouvir. No entanto, não se pode desconsiderar o percentual de usuários que referiu dificuldades neste aspecto, mostrando-se relevante a busca pelos fatores que as determinam, bem como a busca de estratégias que minimizem essas dificuldades.

As dificuldades no manuseio do AASI por idosos é um aspecto também identificado na literatura ${ }^{(5)}$, principalmente na inserção e remoção do molde auricular, utilização de bobina telefônica e memórias do AASI digital; nos AASI analógicos, as dificuldades geralmente envolvem as interferências tecnológicas.

A bobina telefônica serve para melhorar a comunicação ao telefone, tornando a fala mais intensa e inteligível ${ }^{(9)}$. Pesquisadores consideram o não uso do telefone um fator negativo em relação à satisfação do uso da prótese auditiva ${ }^{(10-12)}$. Nesta pesquisa foram encontrados baixos escores referentes ao auxílio do AASI para o uso do telefone, pois nenhum dos indivíduos faz uso da bobina para melhorias da comunicação ao telefone.

A observância aos cuidados com a prótese auditiva para não molhar o circuito interno e não deixá-lo cair ao chão é essencial para a boa conservação da prótese e constitui-se foco de orientações dos fonoaudiólogos aos usuários durante as sessões de adaptação e de reabilitação auditiva, já que a exposição do AASI a água pode provocar oxidação, e as quedas podem danificar o circuito eletrônico da prótese ${ }^{(13)}$.

A limpeza diária é uma das orientações básicas prestadas ao usuário do AASI, que deve ser procedida não somente na 
consulta inicial de uso, mas reforçada em todos os seus retornos ao serviço de saúde auditiva do Sistema Único de Saúde. A sujeira no aparelho pode determinar seu mau funcionamento quando, por exemplo, provoca entupimento da ventilação ou do tubo de silicone do molde, bloqueando a saída do $\operatorname{som}^{(13,14)}$ ou provocar o surgimento de prurido e, posteriormente, infecções no conduto auditivo externo. A presença de gotículas de água no tubo também é um fator característico de má higienização, podendo provocar possível microfonia ${ }^{(13)}$.

Ante essa evidência e a delicadeza das suas implicações para a saúde auditiva dos usuários, cabe investigar os motivos pelos quais estes não realizam a limpeza diária da prótese auditiva, a fim de propor intervenções para reverter tal situação, favorecendo assim a melhoria da qualidade das ações desenvolvidas pelo serviço.

Sabe-se que os moldes são de uso individual e que devem ser lavados diariamente para minimizar os riscos de afecções do conduto auditivo externo. Portanto, o uso compartilhado do molde, associado à falta de higienização diária, aumenta o risco de má adaptação da prótese auditiva.

Importa considerar ainda que portadores de dermatite seborréica ou pele excessivamente oleosa apresentam cerúmen natural do conduto auditivo de forma intensa, que deve ser removido periodicamente ${ }^{(14)}$.

Outro fator determinante pode ser o uso inadequado do molde devido ao desgaste do material e/ou alterações anatômicas. Para confeccionar um novo molde, autores mencionam justificativas como: estar machucando, pequeno ou causando alergias; no caso de adultos, o molde pode alargar um pouco a orelha e tornar-se folgado; molde velho e/ou danificado pelo tempo de uso; alterações nos limiares auditivos ${ }^{(13,14)}$. Aspectos desta natureza podem influenciar na amplificação do som e diminuir a satisfação do usuário.

Em relação à higienização do AASI, o resultado obtido pode ser considerado alarmante quando comparado a outro estudo, no qual evidenciou-se que apenas 3,2\% dos indivíduos referiram não saber realizar a limpeza dos moldes ${ }^{(6)}$.

Os resultados do presente estudo a respeito do uso diário diferem de outras pesquisas ${ }^{(3,7,15)}$ que verificaram uma alta pontuação, superior a quatro pontos, retratando resultados mais positivos para a adaptação à prótese auditiva.

Caso os usuários pesquisados não usem a amplificação sonora por, pelo menos, quatro horas, tempo considerado o mínimo para que haja a adaptação ao mesmo nessa fase inicial ${ }^{(16)}$, o resultado obtido pode ser considerado insatisfatório, já que o maior tempo de uso concorre positivamente para a adaptação. Pesquisas comprovam que após três meses de uso já pode ser percebido o benefício do AASI para a inteligibilidade de fala, principalmente no ruído, desde que os indivíduos usem-no por um tempo igual ou superior a quatro horas ${ }^{(3,15,17)}$.

Alguns fatores podem contribuir para o uso da prótese auditiva por um tempo reduzido. A dificuldade de acesso dos usuários às pilhas para funcionamento das próteses é um dos aspectos presentes em populações de baixa renda, pois a preocupação da carga da pilha acabar e de não se dispor de recurso para comprar uma nova pilha é uma realidade verbalizada pelos participantes.

Além do aspecto financeiro, outros fatores contribuem para o uso reduzido da prótese auditiva, incluindo a dificuldade motora, perceptual em relação à sensação sonora ou relacionada ao conforto, de acordo com a adaptação física ao molde ou à cápsula ${ }^{(1)}$. O desconforto relacionado ao ajuste do molde auricular ao pavilhão auricular ou da cápsula ao canal auditivo externo foi referido em uma pesquisa ${ }^{(6)}$ como um dos fatores que podem causar desconforto, dor e ferimentos no pavilhão auditivo e no conduto auditivo externo após algum tempo de uso.

As dificuldades relacionadas à amplificação sonora também podem ser causas de limitações para uso de prótese, causando, por exemplo, desconforto auditivo para sons de intensidade forte, que podem fazer com que o usuário sinta necessidade de diminuir o volume, alterando o ganho da prótese, ou não fazendo uso constante ${ }^{(1)}$.

A evidência sobre o baixo benefício da amplificação sonora pode ser decorrente do fato de que $75 \%$ dos usuários pesquisados se situam em faixas etárias acima de 61 anos e, com o aumento da idade, as habilidades de reconhecimento de fala vão se deteriorando, devido ao envelhecimento do sistema auditivo, sendo os ambientes ruidosos os que mais comprometem os indivíduos idosos usuários de prótese ${ }^{(17)}$.

Para favorecer o máximo benefício decorrente ao uso da amplificação sonora, estudos ${ }^{(18)}$ concluem que quanto mais tempo o indivíduo utiliza o AASI diariamente, melhor é o seu desempenho comunicativo e, consequentemente, melhor é o relacionamento com as pessoas com quem convive e a troca de informações. Os autores revelam que o tempo de utilização do AASI influencia no benefício oferecido por ele. Em nossa pesquisa, não foi possível comparar a condição prévia ao uso da ampliação com a atual, pois além de não se constituir alvo específico da investigação, os usuários participantes já faziam uso de AASI antes do estudo.

Outro aspecto relevante a ser considerado é que o processo de orientação para os familiares e o usuário de AASI é imprescindível para que este utilize eficazmente seu aparelho, aproveitando ao máximo seus benefícios ${ }^{(19)}$.

A esse respeito, alguns autores ${ }^{(20)}$ mencionam o aconselhamento não só do idoso usuário de AASI, mas também dos familiares, pois assim poderá haver maior compreensão das limitações, atitude positiva frente às dificuldades de comunicação enfrentadas e maior motivação para o uso efetivo do aparelho.

As pessoas que convivem com idosos usuários de AASI necessitam conhecer as limitações da perda auditiva e dos amplificadores sonoros, bem como os meios adequados para compensá-los com ajustes do ouvinte e do falante às estratégias de comunicação ${ }^{(21)}$.

A variável limitação apresentada na Tabela 2 evidencia um baixo índice de satisfação quanto ao uso do AASI, quando comparado ao de outras pesquisas ${ }^{(10,22)}$, nas quais se verificou satisfação elevada. É importante mencionar que a satisfação é medida através da autopercepção de cada usuário, diferenciando-se entre indivíduos, pois varia de acordo com necessidades e expectativas específicas.

A restrição nas atividades cotidianas é uma das condições identificadas em idosos protetizados ${ }^{(23)}$. Pesquisas revelam que a deterioração da função auditiva, que acompanha o processo 
de envelhecimento, afeta tanto a comunicação, quanto os domínios sociais e emocionais, à medida que acarreta uma redução significativa na interação e nos contatos pessoais ${ }^{(24)}$.

Em relação à qualidade de vida dos indivíduos desse estudo, resultados evidenciam que o uso da prótese auditiva está influenciando positivamente, no entanto, observa-se que está prejudicado pelos aspectos de limitação social.

Assim, o relacionamento social do idoso é afetado pelas consequências incapacitantes do envelhecimento, gerando um processo de desvalorização e de diminuição da auto-estima, agravado por sua dificuldade de comunicação ${ }^{(25)}$, reduzindo portanto, a qualidade de vida do indivíduo com limitações sociais. Estudos evidenciam, de forma clara, as dificuldades no âmbito social vivenciadas por idosos usuários de $\mathrm{AASI}^{(8,24)}$. A evidência de dificuldades de comunicação, mesmo com o uso de prótese, observada no presente estudo indica que o AASI por si só não atinge um de seus mais importantes objetivos, ou seja, facilitar a comunicação, contribuindo para a melhoria do convívio social.

Alguns autores ${ }^{(21,26)}$ consideram que o treinamento auditivo ajuda os indivíduos em fase de adaptação de AASI a melhorar as habilidades de reconhecimento de fala, inclusive, aquelas em que modificações espontâneas são observadas no decorrer do tempo. Considera-se ainda que esse processo ajuda a retirar o indivíduo do isolamento, possibilitando melhor qualidade de vida ${ }^{(27)}$. Dessa forma, alguns pesquisadores ${ }^{(7,28,29)}$ sugerem a aplicação do questionário IOI-HA durante o processo de reabilitação para auxiliar nas possíveis orientações para o uso do AASI.

Reconhecer a necessidade da proposição de ações de educação em saúde é inicialmente compreender que essas não se restringem somente a orientações sobre cuidados e manuseio de prótese auditiva durante as consultas iniciais e de retorno. É de substancial importância entender que a educação em saúde envolve elementos essenciais de aprendizagem que gerem motivação para o desenvolvimento de habilidades e de mudanças na trajetória de vida, tendo como base a participação e o diálogo $\mathrm{o}^{(30)}$.

Nessa perspectiva, os usuários de AASIs participarão de um processo de capacitação contínuo, em que deverão sentir-se motivados e responsáveis por cuidar da sua própria saúde.

\section{CONCLUSÃO}

Os usuários de AASI assistidos pelo SUS, participantes deste estudo, apresentam dificuldades em relação ao seu uso e manuseio, que influenciam negativamente na adaptação ao mesmo. Estas dificuldades interferem no uso diário da amplificação sonora.

Os resultados obtidos mostram a importância da promoção de ações de educação em saúde auditiva, auxiliando no planejamento de estratégias e na elaboração de materiais educativos que favoreçam a capacitação dos usuários a fim de minimizar as dificuldades de adaptação.

\begin{abstract}
Purpose: To evaluate the adaptation to hearing aids by users attended by the Brazilian public health system (SUS), and to propose educational actions, based on the users' needs. Methods: Descriptive quantitative research, which evaluated 32 adults with hearing loss during the adaptation of hearing aids provided by the Unified Health System (SUS). Two structured questionnaires with closed questions were applied, and the data received descriptive analysis. Results: Moderate limitations and restrictions were observed, as well as reduced time of daily use, limiting the benefit of amplification. Regarding use and handling, it was observed that $70.8 \%$ put on and take off the device by themselves, $79.2 \%$ take it off to bathe, $87.5 \%$ do not perform daily hygiene, and $37.5 \%$ have allergies or pain attributed to the use of the mold. Conclusion: Users present difficulties in the use and handling of hearing aids, which negatively influence their adaptation to it. Actions to promote hearing health are necessary, with the aim to minimize these difficulties and facilitate hearing aid adaptation.
\end{abstract}

Keywords: Hearing; Hearing aids; Adaptation; Hearing loss; Health education; Unified Health System

\section{REFERÊNCIAS}

1. Braga, SRS Conhecimentos essenciais para atender bem o usuário com prótese auditiva. São José dos Campos: Pulso, 2003. Considerações básicas sobre o processo de indicação, seleção e adaptação de próteses auditivas. p.11-5.

2. Brasil. Ministério da Saúde. Política nacional de saúde da pessoa portadora de deficiência. Brasília: Ministério da Saúde; 2009.

3. Prates LP, Iório MC. Aclimatização: estudo do reconhecimento de fala em usuários de próteses auditivas. Pró-Fono. 2006;18(1):259-66.

4. Ferreira MI, Sant' Anna IM. Conhecimento de usuários de aparelhos auditivos sobre o processo de adaptação. Arq Int Otorrinolaringol. 2008;12(3):384-92.
5. Campos K, Oliveira JRM, Blasca WQ. Processo de adaptação de aparelho de amplificação sonora individual: elaboração de um DVD para auxiliar a orientação a indivíduos idosos. Rev Soc Bras Fonoaudiol. 2010;15(1):19-22.

6. Freitas CD, Costa MJ. Processo de adaptação de próteses auditivas em usuários atendidos em uma instituição pública federal - parte I: resultados e implicações com o uso da amplificação. Rev Bras Otorrinolaringol. 2007;73(6):744-51.

7. Cox RM, Alexander GC. The international outcome inventory for hearing aids (IOI-HA): psychometric properties of the English version. Int J Audiol. 2002;41(1):30-5. 
8. Vieira EP, Miranda EC, Calais II, Carvalho IM, Iório MC, Borges AC. Proposta de acompanhamento em grupo para idosos protetizados. Rev Bras Otorrinolaringol. 2007;73(6):752-8.

9. Kobata DY. Componentes das próteses auditivas. In: Braga SRS. Conhecimentos essenciais para atender bem o usuário com prótese auditiva. São José dos Campos: Pulso, 2003. p.17-22.

10. Veiga IR, Merlo ARC, Mengue SS. Satisfação com a prótese auditiva na vida diária em usuários do sistema de saúde do exército. Rev Bras Otorrinolaringol. 2005;71(1):67-73.

11. Paiva KM, Maciel PM. Compreendendo o idoso usuário de próteses auditivas. Cienc Saúde Coletiva (online). 2011;16(6): 2927-34.

12. Lessa AH, Costa MJ, Becker KT, Vaucher AV. Satisfação de usuários de próteses auditivas, com perda auditiva de graus severo e profundo. Arq Int Otorrinolaringol. 2010;14(3):338-45.

13. OTE: On the ear. Manual de operações. Starkey Laboratorios; 2008.

14. Almeida K, Taguchi, CK. Moldes auriculares: considerações estruturais e acústicas. In: Almeida K, Iório MC. Prótese auditiva: fundamentos teóricos e aplicações clínicas. 2a ed. São Paulo: Lovise, 2003. p.189215.

15. Costa IP, Iório MCM.Próteses auditivas: avaliações objetivas e subjetivas em usuários de amplificação linear e não-linear. Pró-Fono. 2006;18(1):21-30.

16. Almeida K. Avaliação dos resultados da intervenção. In: Almeida K, Iório MC. Prótese Auditiva: fundamentos teóricos e aplicações clínicas. 2a ed. São Paulo: Lovise, 2003. p. 335-55.

17. Bucuvic EC, Iório MC. Benefício e dificuldades auditivas: um estudo em novos usuários de próteses auditivas após dois e seis meses de uso. Fono Atual. 2004;29(7):19-29.

18. Miranda EC, Calais II, Vieira EP, Carvalho IM, Borges AC, Iorio MC. Dificuldades e benefícios com o uso de prótese auditiva: percepção do idoso e sua família. Rev Soc Bras Fonoaudiol. 2008;13(2):166-72.

19. Creppe SV, Blasca WQ, Oliveira JR. Análise da efetividade de um programa de adaptação do aparelho de amplificação sonora individual para indivíduos deficientes auditivos idosos. J Bras Fonoaudiol. 2005;5(22):284-90.
20. Ferrari DV, Blasca WQ, Coube CZ. Conceitos básicos e características de tecnologia dos aparelhos de amplificação sonora individual. In: Bevilacqua MC, Moret AL. Deficiência auditiva: conversando com familiares e profissionais da saúde. São José dos Campos: Pulso; 2005. p.94-122.

21. Musiek FE, Lee WW. Reabilitação auditiva do idoso. In: Musiek FE, Rintelmann WF. Perspectivas atuais em avaliação auditiva. Barueri: Manole; 2001. p.239-67.

22. Teixeira CF, Augusto LG, Caldas Neto SS. Prótese auditiva: satisfação do usuário com sua prótese e com seu meio ambiente. Rev CEFAC. 2008;10(2):245-53.

23. Assayag FH, Russo IC. Avaliação subjetiva do benefício e dos efeitos proporcionados pelo uso de amplificação sonora em indivíduos idosos. Disturb Comun. 2006;18(3):383-90.

24. Pinheiro MM, Pereira ID. Processamento auditivo em idosos: estudo da interação por meio de testes com estímulos verbais e não-verbais. Rev Bras Otorrinolaringol. 2004;70(2):209-14.

25. Quintero SM, Marotta RM, Marone SA. Avaliação do processamento auditivo de indivíduos idosos com e sem presbiacusia por meio do teste de reconhecimento de dissílabos em tarefa dicótica - SSW. Rev Bras Otorrinolaringol. 2002;68(1):28-33.

26. Petry T, Santos SN, Costa MJ. Efeito da aclimatização em novos usuários de próteses auditivas lineares e não lineares. Rev Soc Bras Fonoaudiol. 2010;15(4):526-32.

27. Freire KG. Adaptação de prótese auditiva em idosos. In: Braga SR. Conhecimentos essenciais para atender bem o usuário com prótese auditiva. São José dos Campos: Pulso; 2003. p.67-79.

28. Gasparin M, Menegotto IH, Cunha CS. Propriedades psicométricas do questionário internacional: aparelho de amplificação sonora individual. Braz J Otorhinolaryngol. 2010;76(1):85-90.

29. Heuermann H, Kinkel M, Tchorz J. Comparison of psychometric properties of the International Outcome Inventory for Hearing Aids (IOI-HA) in various studies. Int J Audiol. 2005;44(2):102-9.

30. Ribeiro LM, Rasera EF. Os idosos e o uso de próteses auditivas: identificando os repertórios interpretativos que justificam essa decisão. Psicol Soc. 2008;20(3):425-33. 